\title{
Antibacterial and Ciprofloxacin-Potentiation Activities of Cinnamomum zeylanicum Extracts against Some Pathogenic Bacteria
}

\author{
Azizollah Ebrahimi (PhD) \\ Department of Pathobiology, School \\ of Veterinary Science, Shahrekord \\ University, Shahrekord, Iran \\ Azimeh Babaaie (DVM Student) \\ DVM Student of Pathobiology \\ Department, Veterinary College, \\ Shahrekord University, Iran \\ Mojtaba Boniadian (PhD) \\ Department of Public Health, School \\ of Veterinary Science, Shahrekord \\ University, Shahrekord, Iran \\ Sharareh Lotfalian (MSc) \\ Department of Pathobiology, School of \\ Veterinary Science, Shahrekord \\ University, Shahrekord, Iran \\ Corresponding Author: Azizollah \\ Ebrahimi \\ Email: A_kahrizsangi@yahoo.com \\ Tel: +98-9133197980 \\ Address: Department of Pathobiology, \\ School of Veterinary Science, \\ Shahrekord University, Shahrekord, \\ Iran \\ Received: 21 May 2018 \\ Revised: 07 Dec 2018 \\ Accepted: 19 Dec 2018 \\ cc) (i) (8)
}

This work is licensed under a Creative Commons Attribution 4.0 License.

\begin{abstract}
Background and Objectives: Efflux-based systems may play a role in resistance to fluoroquinolones in Gram-negative pathogenic bacteria. Extracts of some medicinal plants contain molecules that can act as efflux pumps inhibitors. In this study, we aimed to evaluate antibacterial activities of ethanolic and chloroform extracts of Cinnamomum zeylanicum and their possible synergistic activity with ciprofloxacin against some Gram-negative pathogenic bacteria. We also analyzed the extracts for presence of efflux pump inhibitors against the examined bacteria.

Methods: Powdered dried leaves and branches of $C$. zeylanicum were extracted with ethanol (85\%) and chloroform by the maceration method. Minimum inhibitory concentrations of the extracts alone or combined with ciprofloxacin and phenylalaninearginine $\beta$-naphthylamide (an efflux pump inhibitor) were determined against Pseudomonas aeruginosa, Acinetobacter bummani, Escherichia coli and Salmonella enteritidis using the double serial microdilution method.

Results: The extracts of $C$. zeylanicum inhibited the growth of all studied bacteria. Synergistic effects were noted between the extracts and ciprofloxacin against all tested bacteria other than P. aeruginosa. Ciprofloxacin efflux pumps in E. coli, S. enteritiditis and A. baumannii were inhibited by the extracts of $C$. zeylanicum.

Conclusion: The extracts of $C$. zeylanicum could be used as ciprofloxacinpotentiating agents against some Gram-negative pathogens.
\end{abstract}

Keywords: Anti-bacterial agents, Cinnamomum zeylanicum, Bacterial pathogens, Efflux pumps.

This paper should be cited as: Ebrahimi A, Babaaie A, Boniadian M, Lotfalian SH [Antibacterial and CiprofloxacinPotentiation Activities of Cinnamomum zeylanicum Extracts against Some Pathogenic Bacteria]. mljgoums. 2019; 13(3):6- 10 


\section{INTRODUCTION}

Since bacterial efflux pumps can decrease antibiotics concentration inside bacterial cells and thereby increase resistance to antimicrobials, recent studies have taken an interest in discovering safe inhibitors of these pumps.

Multidrug resistant Gram-negative bacteria utilize multiple mechanisms including reduced outer membrane permeability and active efflux against numerous antimicrobials (1). Recent studies show that efflux-based systems may play a role in resistance of Gram-negative pathogens to fluoroquinolones (2). Overexpression of these pumps in pathogenic bacteria results in the emergence of ciprofloxacin resistance $(2,3)$.

Phenylalanine-arginine $\quad \beta$-naphthylamide $(\mathrm{PABN})$ is a known efflux pump inhibitor (EPI) (4) that selectively inhibits the activity of a broad range of efflux pumps such as MexAB-OprM, MexEF-OprN, MexCD-OprJ and MexXY-OprM in Pseudomonas aeruginosa and AcrAB-TolC in some Enterobacteriaceae species (5).

Many medicinal plants have been traditionally used in various parts of the world including Iran for the treatment of infections caused by pathogenic bacteria $(6,7)$. It is reported that extracts of some medicinal plants contain molecules that can act as EPIs for bacteria (8). Cinnamomum zeylanicum is a well-known medicinal herb that is commonly used in foods for its medicinal properties (9).

In this study, we examine the antibacterial activity of ethanolic and chloroform extracts of C. zeylanicum combined with ciprofloxacin against some Gram-negative pathogenic bacteria. We also analyzed the extracts for presence of EPI for the examined bacteria.

\section{MATERIALS AND METHODS}

Dried leaves and branches of $C$. zeylanicum were obtained from local markets of Shahrekord, Iran. After grinding, the plant extracts were prepared through maceration. Briefly, powdered plants in conical flasks were mixed with ethanol $(85 \%)$ and chloroform at room temperature for 2-3 days. Daily filtration and addition of fresh solvents were done for ethanol extraction, while one-stage filtration was carried out for chloroform extraction. The obtained filtrates were evaporated by incubation at $34{ }^{\circ} \mathrm{C}$ for the ethanol and under biolaminar safety hood for the chloroform extracts. The evaporated extracts were kept in a refrigerator for future use (10).

Reference strains of $P$. aeroginosa ATCC 9027, Acinetobacter baumannii, NCTC 13305, Escherichia coli ATCC 25922 and Salmonella enteritidis RTCC 2465 were used for assays. The strains were first grown in Lauria Bertani broth (Biomark, India) in a refrigerator and then sub-cultured on suitable media 24 hours prior to antimicrobial testing. In all antibacterial tests, Mueller Hinton broth (Merck, Germany), ciprofloxacin (SigmaAldrich) and PA $\beta N$ (Sigma-Aldrich) were used as medium, microbial growth indicator and an EPI, respectively.

Minimum inhibitory concentrations (MICs) of the extracts and ciprofloxacin were determined via tube dilution test. MICs of drug combinations were determined by double serial microdilution method according to the Clinical and Laboratory Standards Institute guidelines (11). In brief, the examined bacteria were grown at $37{ }^{\circ} \mathrm{C}$ for $18-24$ hours. Culture turbidity was adjusted to 0.5 McFarland $\left(1.5 \times 10^{8} \mathrm{CFU} / \mathrm{ml}\right)$ and the cultures were diluted to obtain an inoculum of $5 \times 10^{5}$ CFU/well. Four MIC of drug/drugs were inoculated into the first wells of 96-well microplates, followed by double dilution in subsequent wells. The two last wells were used for the positive and negative controls. The well-plates were placed on a shaking incubator for 18 hours at $37{ }^{\circ} \mathrm{C}$. Lowest concentration that inhibited the growth of bacteria was defined as the MIC. To evaluate synergistic activity of ciprofloxacin with the ethanolic and chloroform extracts, antibacterial activity of ciprofloxacin-extract combinations was compared with that of ciprofloxacin-PABN (30 $\mu \mathrm{g} / \mathrm{ml})$. Synergistic activity of drug combinations was calculated as the $\mathrm{MIC}_{\text {Antibiotic }}$ combination $/ \mathrm{MIC}_{\text {Antibiotic alone }}$ ratio and the results were interpreted as follows: synergy $(<0.5)$, indifferent ( 0.5 to 4$)$ and antagonism (> 4) (12, 13). All assays were performed in duplicate. Effect of drug combinations on efflux pumps in synergistic cases was interpreted according to a method described by Martins et al. (14) with slight modifications. Briefly, the examined strains were cultured on Mueller Hinton agar (MHA, Merck, Germany). After creating four wells in the medium, $50 \mu \mathrm{l}$ ethidium bromide (EB, $6 \mu \mathrm{g} / \mathrm{mL}$ ) and $50 \mu \mathrm{l}$ distilled water were inoculated into one well. 
Synergistic ethanolic or chloroform extracts along with EB were inoculated into two other wells, and $\mathrm{Pa} \beta \mathrm{N}+\mathrm{EB}$ (1 MIC concentration and $50 \mu \mathrm{L}$ volume for each case) was inoculated in the last well. After overnight incubation at $37^{\circ} \mathrm{C}$, accumulation of EB in the presence of an EPI such as PABN indicated efflux inhibition (detected by fluorescence from the excitation of EB by UV light) (14).

\section{RESULTS}

The chloroform and ethanolic extracts of C. zeylanicum in combination with ciprofloxacin were examined for possible synergistic and EPI activities. The MICs of the ethanolic and chloroform extracts and drug combinations against the examined bacteria are presented in table 1 . The chloroform extract showed the best antibacterial activity against the examined bacterial strains. When investigating the MICs, the ethanolic extract of C. zeylanicum showed synergistic activity with ciprofloxacin against $S$. enteritidis and $E$. coli, while for the chloroform extract, a synergistic activity was observed against $A$. bumannii and $S$. enteritidis (Table 1). Increased amount of EB accumulation in the presence of an EPI, such as PA $\beta N$ indicates efflux inhibition (12). To clarify whether the synergistic activity with ciprofloxacin exhibited by the extracts of $C$. zeylanicum was due to efflux inhibition, the accumulation of EB in bacterial cells in the presence and absence of 1 MIC of each extract was evaluated. In all examined synergistic cases, the extracts of $C$. zeylanicum increased the amount of EB accumulation (i.e. reduced efflux). In the case of $P$. aeruginosa and $A$. baumanni, the ethanolic extract reduced the MIC of ciprofloxacin but PA $\beta \mathrm{N}$ did not (Table 1).

Table 1- MICs $(\mu \mathrm{g} / \mathrm{mL})$ of ciprofloxacin and PAßN against some Gram-negative bacteria in the absence and presence of $C$. zeylanicum extracts

\begin{tabular}{|c|c|c|c|c|c|c|c|}
\hline $\begin{array}{l}\text { Combination } \\
\text { Bacteria }\end{array}$ & Cip. & Eth.E.+ Cip & Eth.E. & Ch.E. +Cip. & Ch.E. & $\mathbf{P A \beta N}$ & PAßN+Cip \\
\hline P. aeroginosa & 1.98 & 0.992 & 3120 & 1.978 & 3125 & 3.75 & 1.98 \\
\hline S. enteritidis & 0.0312 & 0.0078 & 100000 & 0.0078 & 25000 & 7.5 & 0.0156 \\
\hline $\begin{array}{c}\text { E. coli } \\
\text { A. baumannii }\end{array}$ & $\begin{array}{c}0.0156 \\
8\end{array}$ & $\begin{array}{c}0.0039 \\
2\end{array}$ & $\begin{array}{c}50000 \\
3120\end{array}$ & $\begin{array}{c}0.0156 \\
0.25\end{array}$ & $\begin{array}{c}3120 \\
25000\end{array}$ & $\begin{array}{c}7.5 \\
3.75\end{array}$ & $\begin{array}{c}0.0039 \\
8\end{array}$ \\
\hline
\end{tabular}

* Synergistic activities are shown as bold numbers.

*Eth.E., Ch. E. and Cip stand for ethanolic extract, chloroform extract and ciprofloxacin, respectively.

\section{DISCUSSION}

Nowadays, it is crucial to discover novel approaches and antimicrobials to combat antibiotic resistance and treat resistant bacterial infections. In this regard, identification of natural compounds with efflux pump inhibitory properties is considered a promising approach $(15,18)$. In the present study, we examined the antibacterial and efflux pump inhibitory activities of $C$. zeylanicum extracts.

It is reported that the bacterial strains examined in our study contain multidrug resistance efflux pumps $(2,5)$. It appears that the extracts of $C$. zeylanicum inhibited the growth of all tested bacterial strains within a concentration range of 3120 to $100000 \mu \mathrm{g} / \mathrm{mL}$. The best antibiotic-potentiation activity with ciprofloxacin was observed against $S$. enteritidis and $E$. coli by the ethanolic extract and against $S$. enteritidis and A. baumanni by the chloroform extract. These results confirm that the extracts of $C$. zeylanicum contain compounds with efflux pump inhibitory activity.

There are many bacterial efflux pumps that expel ciprofloxacin out of the cell, and several studies have shown that plant extracts could act as EPI against these pumps $(1,8)$. In all examined synergistic cases, the extracts of $C$. zeylanicum increased the amount of EB accumulation (i.e. reduced efflux).

Evidence suggests that extract of other plants may also c ontain compounds with efflux pump inhibitory or antibacterial activities $(16,18)$. A study reported that essential oil from a Corsican plant, Helichrysum italicum, could reduce the MIC of chloramphenicol against Enterobacter aerogenes, A. baumannii and P. aeruginosa (16). The ethanolic extract combined with 
ciprofloxacin showed significantly greater antibiotic activity against $S$. enteritidis, $P$. aeroginosa and $A$. baumannii compared with the Pa $\beta \mathrm{N}$-ciprofloxacin combination (Table 1). This shows that at least one active compound from this plant, acting inside the bacterial cell could be the powerful substrate of efflux pumps of the mentioned bacteria.

Other studies demonstrated the synergistic effects of $C$. zeylanicum extracts with different antibiotics on other bacteria, thus suggesting that some constituents of these extracts can act as an EPI (18).

The MIC of ciprofloxacin against $P$. aeroginosa and $A$. baumannii decreased when used in combination with the extracts but not with PA $\beta N$. This indicates that these extracts may also act by damaging cell membrane or cell wall of the bacteria and thereby facilitate the penetration of ciprofloxacin into bacterial cell $(19,20)$.

The presence of AcrB efflux pumps confers $\mathrm{PA} \beta \mathrm{N}$ resistance (21), which may indicate that the strains of $P$. aeroginosa and A. baumannii tested in our study overexpress AcrB efflux pumps. However, it is suggested to further investigate active constituents and phytochemica propertie of $C$. zeylanicum extracts for elucidating the

\section{REFERENCES}

1. Fankam AG, Kuiate JR, Kuete V. Antibacterial and antibiotic resistance modifying activity of the extracts from allanblackia gabonensis, combretum molle and gladiolus quartinianus against Gram-negative bacteria including multi-drug resistant phenotypes. BMC Complement Altern Med. 2015; 15(1): 260. doi: 10.1186/s12906-015-0726-0.

2. Adabi M, Talebi-Taher M, Arbabi L, Afshar M, Fathizadeh S, Minaeian S, et al. Spread of efflux pump over expressing-mediated fluoroquinolone resistance and multidrug resistance in Pseudomonas aeruginosa by using an efflux pump inhibitor. Infect Chemother. 2015; 47(2): 98-104. doi: 10.3947/ic.2015.47.2.98.

3. Blot S, Depuydt P, Vandewoude K, De Bacquer D. Measuring the impact of multidrug resistance in nosocomial infection. Curr Opin Infect Dis. 2007; 20(4): 391-6.

4. Lamers RP, Cavallari JF, Burrows LL. The efflux inhibitor phenylalanine-arginine beta-naphthylamide $(P A B N)$ permeabilizes the outer membrane of gramnegative bacteria. PLoS One. 2013; 8(3): e60666.

5. Dreier J, Ruggerone P. Interaction of antibacterial compounds with RND efflux pumps in Pseudomonas aeruginosa. Front Microbiol. Front Microbiol. 2015; 6: 660. doi: 10.3389/fmicb.2015.00660. possiblemechanisms of antibiotic potentiation and antimicrobial activities.

\section{CONCLUSION}

Combination of the extracts of $C$. zeylanicum and ciprofloxacin could be effective for treatment of infections caused by the tested bacterial strains.

\section{ACKNOWLEDGMENTS}

The authors appreciate all individuals who willingly participated in the study. This study was derived from a thesis performed by Azimeh Babaie for completion of a doctorate degree in veterinary medicine.

\section{Authors' Contributions}

Azizollah Ebrahimi designed, supervised and wrote the manuscript. Azimeh Babaie carried out the examinations, analyzed the results and wrote parts of the manuscript. Mojtaba Boniadian supervised the study and Sharareh Lotfalian supervised the examinations.

\section{CONFLICT OF INTEREST}

There is no conflict of interest regarding the publication of this article.

6. Jalali H, Nejad AM, Ebadi AG, Laey G. Ethnobotany and lk pharmaceutical properties of major trees or shrubs in northeast of Iran. Asian Journal of Chemistry. 2009; 21(7): 5632-8.

7. Nejad AS, Kamkar A, Giri A, Pourmahmoudi AA. Ethnobotany and folk medicinal uses of major trees and shrubs in Northern Iran. J Med Plants Res. 2013; 7(7): 284-9.

8. Stavri M, Piddock LJ, Gibbons S. Bacterial efflux pump inhibitors from natural sources. $\mathrm{J}$ Antimicrob Chemother. 2007; 59(6): 1247-60.

9. WONg YC, Ahmad-Mudzaqqir MY, Wan-Nurdiyana WA. Extraction of essential oil from Cinnamon ( $C$. zeylanicum). Orient J Chem. 2014; 30(1): 37-47.

10. Azwanida N. A Review on the extraction methods use in medicinal plants, principle, strength and limitation. $\mathrm{J}$ Med Aromat Plants. 2015; 4: 196. doi:10.4172/21670412.1000196.

11. CLSI. Methods for dilution antimicrobial susceptibility tests for bacteria that grow aerobically; approved standard, Ninth Edition. CLSI document M07A9. Wayne, PA: Clinical and Laboratory Standards Institute; 2012. 
12. Shahverdi A, Monsef-Esfahani H, Tavasoli F, et al. Trans-Cinnamaldehyde from $C$. zeylanicum bark essential oil reduces the clindamycin resistance of Clostridium difficile in vitro. J Food Sci. 2007; 72(1): S055-S8.

13. Braga LC, Leite AA, Xavier KG, Takahashi JA, Bemquerer MP, Chartone-Souza E, et al. Synergic interaction between pomegranate extract and antibiotics against Staphylococcus aureus. Can J Microbiol. 2005; 51(7): 541-7.

14. Martins M, McCusker MP, Viveiros M, Couto I, Fanning S, Pagès JM, et al. A simple method for assessment of MDR bacteria for over-expressed efflux pumps. Open Microbiol J. 2013; 7: 72-82. doi: $10.2174 / 1874285801307010072$.

15. Tegos G, Stermitz FR, Lomovskaya O, Lewis K. Multidrug pump inhibitors uncover remarkable activity of plant antimicrobials. Antimicrob Agents Chemother. 2002; 46(10): 3133-41. doi: 10.1128/AAC.46.10.31333141.2002.

16. Lorenzi V, Muselli A, Bernardini AF, Berti L, Pagès JM, Amaral L, et al. Geraniol restores antibiotic activities against multidrug-resistant isolates from gramnegative species. Antimicrob Agents Chemother. 2009; 53(5): 2209-11. doi: 10.1128/AAC.00919-08.
17. Piddock LJ, Garvey MI, Rahman MM, Gibbons S, et al. Natural and synthetic compounds such as trimethoprim behave as inhibitors of efflux in Gramnegative bacteria. J Antimicrob Chemother. 2010; 65(6): 1215-23. doi: 10.1093/jac/dkq079.

18. Garvey MI, Rahman MM, Gibbons S, Piddock LJ. Medicinal plant extracts with efflux inhibitory activity against Gram-negative bacteria. Int J Antimicrob Agents. 2011; 37(2): 145-51. doi: 10.1016/j.ijantimicag.2010.10.027.

19. Karsha PV, Lakshmi OB. Antibacterial activity of black pepper (Piper nigrum Linn.) with special reference to its mode of action on bacteria. Indian J Nat Prod Resour. 2010; 1(2): 213-5.

20. Opperman TJ, Nguyen ST. Recent advances toward a molecular mechanism of efflux pump inhibition. Front Microbiol. 2015; 6: 421. doi: 10.3389/fmicb.2015.00421. 21. Ohene-Agyei T, Mowla R, Rahman T, Venter H. Phytochemicals increase the antibacterial activity of antibiotics by acting on a drug efflux pump. MicrobiologyOpen. $2014 ; \quad 3(6)$ : 885-96. doi: $10.1002 / \mathrm{mbo} 3.212$. 